\title{
Kinderarmut - Eine komplexe Herausforderung für staatliches Handeln
}

In Deutschland wächst eine große Gruppe von Kindern unter Armutsbedingungen auf. Sie erfahren bereits im frühsten Kindesalter vielfältige Mangelsituationen, die ihre Entwicklungs- und Zukunftschancen komplex beeinträchtigen. Bereits Mitte der 1990er Jahre formulierte Hauser warnend den Begriff der „Infantilisierung der Armut“. Heute ist dies ein verfestigtes Phänomen. Dennoch besteht mittlerweile weniger ein wissenschaftliches Erkenntnisdefizit über Armut als ein hohes Umsetzungsdefizit bei der Armutsprävention, die, selbst wenn Armut eintritt, die individuellen Folgen für die kulturelle, soziale und gesundheitliche Lage der Kinder mindern.

\section{Armut von Kindern - Noch immer ein Tabuthema?}

Ein Tabuthema hat den Raum wissenschaftlicher Diskurse verlassen und ist ins Zentrum von (fach-)politischen und besonders öffentlichen Diskussionen gerückt: Immer mehr Kinder wachsen unter Armutsbedingungen in Deutschland auf. Die Folgen zeigen sich allerorts mehr und mehr. Kontroversen gibt es über die Ursachen und Verantwortlichkeiten: Geht es um die Bewältigung individueller „Notlagen" von Kindern, so wird dies meist als Versagen der Eltern angesehen und mehr Elternverantwortung eingefordert. Geht es um monetäre Verteilungs- und soziale Gerechtigkeitsfragen sowie ganz besonders um die Sicherung der generellen $\mathrm{Zu}$ kunftsfähigkeit unserer Gesellschaft, so wird dies meist als Versagen des Sozialstaates bewertet. Ein Defizit, dem mit mehr öffentlicher Verantwortung für alle Kinder begegnet werden sollte. Der Zwölfte Kinder- und Jugendhilfebericht der Bundesregierung (BMFSFJ 2005a) hat den untrennbaren Zusammenhang zwischen beidem deutlich herausgestellt: Kinder - und damit sind alle, auch arme, gemeint - erfordern eine private und eine öffentliche Verantwortung.

Ein solches Verständnis, das nicht auf Schuldzuschreibungen beruht, sondern auf Verantwortungsübernahme mit jeweils eigenen Ausprägungen durch eine Vielzahl unterschiedlicher Akteure setzt, ist ein konstruktiver Zugang, um Armut bei Kindern zu erfassen und Armutsprävention für Kinder in die Tat umzusetzen. Hierfür werden folgende Fragen mittels theoretischer Klärungen und empirischer Daten- befunde erläutert: Wie verbreitet ist Kinderarmut (Abschnitt 2)? Wie entsteht sie (Abschnitt 3)? Was ist mit dem Begriff „Kinderarmut" über Einkommensarmut hinaus verbunden (Abschnitt 4)? Welche konkreten Folgen zeigen sich in der Kindheit bis zum Ende der Grundschulzeit (Abschnitt 5)? Wie kann Armut bewältigt werden und welche Rolle spielen Staat und andere Akteure (Abschnitt 6-8)?

\section{Jedes vierte Kind wächst in Armut auf}

Um einen realistischen Anhaltspunkt über den Umfang der Kinderarmut zu erhalten, lassen sich zum einen Zahlen über die verschiedenen Gruppen von SozialtransferBeziehern und zum anderen über potenzielle Leistungsberechtigte heranziehen. Auf dieser Basis ist davon auszugehen, dass 3 Mio. der unter 15-Jährigen von Armut betroffen sind:

- Rund 1,84 Mio. unter 15-Jährige erhielten im November 2007 Sozialgeld gemäß SGB II (Bundesagentur für Arbeit 2007);

- rund 19.000 unter 15-Jährige bezogen im Jahr 2005 Sozialhilfe gemäß SGB XII (Statistisches Bundesamt 2007, S. 213-215); - rund 64.500 unter 15-Jährige erhielten im gleichen Jahr Leistungen gemäß Asylbewerberleistungsgesetz (Statistisches Bundesamt 2007, S. 213-215);

- weiterhin lebten 2007 rund 150.000 Kinder in Familien, die Anspruch auf Kinderzuschlag haben (Deutscher Bundestag 2007, S. 2); 1

- schließlich ist eine Dunkelziffer beim ALG II-Bezug, die nach neuster Berechnung bei $48 \%$ liegt (Becker 2006), anzu- nehmen. Die Größenordnung liegt hier bei rund 0,9 Mio. der unter 15-Jährigen.

Die regionale Verteilung weist auf ein OstWest-, ein Nord-Süd- sowie ein StadtLand-Gefälle hin, wie die ALG II-Bezugsdaten belegen. In Westdeutschland lebten im März 2007 rund 14,3 \% und in Ostdeutschland $31 \%$ der unter 15-Jährigen von ALG II. Aber auch innerhalb einer Region oder eines Bundeslandes sind höchst unterschiedliche Risikoverteilungen gegeben. Zum gleichen Zeitpunkt (03/2007) lag die ALG-II Quote in Bayern bei 8,5 \%, dagegen in Nürnberg bei $23,5 \%$ der unter 15-Jährigen (Bundesagentur für Arbeit 2007; Schröder 2007).

\section{Kinderarmut ist primär strukturell bedingt}

Als die drei wesentlichen Ursachen für Armut von Erwachsenen in Deutschland gel-

\footnotetext{
1 Die Zahlenzusammenstellung liefert die bei Verfassung des Aufsatzes aktuellsten verfügbaren Daten für die verschiedenen Gruppen mit Transferleistungsbezug. Die Zahlen bilden Anhaltswerte, um eine ungefähre Größenordnung über die tatsächliche Betroffenheit zu erhalten. Eine amtliche Gesamtzahl - zeitnah zu einem Stichtag lässt sich nicht benennen.
}

Gerda Holz, Politikwissenschaftlerin, Sozialarbeiterin grad. am Institut für Sozialarbeit und Sozialpädagogik, Frankfurt/Main. Arbeitsschwerpunkte: Armut und Soziale Ausgrenzung, Soziale Infrastrukturentwicklung. e-mail: gerda.holz@iss-ffm.de 
ten (Langzeit-)Erwerbslosigkeit sowie Erwerbstätigkeit mit geringer Arbeitszeit und mit Niedriglohn. Letzteres, also Armut trotz Arbeit („working poor“), nimmt rasant an Umfang und Bedeutung zu. Eine weitere Ursache sind die Sozialreformen (besonders Hartz IV), die mit einer Verringerung von Sicherungsansprüchen einhergehen (DIW 2007). Ein nur selten thematisierter Ursachenkomplex ist nicht oder nur gering entlohnte Haus-, Pflege-, Erziehungs- und Sorgearbeit, von der vor allem Frauen betroffen sind. Darüber hinaus können Überschuldung, Scheidung oder Trennung, Behinderungen oder multiple soziale Problemlagen ursächlich sein. Kinder, die in Haushalten mit einem oder mehreren der genannten sozialen Merkmale aufwachsen, sind besonders armutsgefährdet.

Neben diesen strukturellen Aspekten hat das individuelle Verhalten der Eltern allgemein Einfluss auf die Lebenslagen von (armen) Kindern. Die längsschnittliche AWO-ISS-Studie zu Kinderarmut (Hock et al. 2000; Holz et al. 2006) belegte bereits 2000 und erneut 2004/05, dass arme und nicht-arme Eltern gleichermaßen in ihrem Erziehungsverhalten und ihren Erziehungsstilen, aber auch im Bewältigungshandeln, höchst individuell und damit unterschiedlich sind, d.h. Armut an sich determiniert das Erziehungsverhalten nicht. Auch arme Eltern wollen das Beste für ihr Kind, erhoffen sich positive Zukunftsperspektiven und versuchen, negative Folgen des Ressourcenmangels aufzufangen (Meier et al.2003; Walper 2005). Das bestätigt eine im Herbst 2007 von Wüstendörfer durchgeführte Studie bei armutsbetroffenen Familien in Nürnberg. Sie belegt zweierlei: (a) Elterliches Bewältigungshandeln armer Mütter/Väter führt zu einem breiten Spektrum möglicher kindlicher Lebenslagen, von keinerlei bis hin zu häufigen Einschränkungen. (b) Arme Eltern sparen zunächst bei sich selbst und bei den gemeinsamen Familienaktivitäten, bevor es zuletzt zu Einschränkungen in der kindlichen Versorgung kommt. ${ }^{2}$

Nicht alle Bevölkerungsgruppen sind gleichermaßen von Armut betroffen, sondern es gibt gruppenbezogen unterschiedliche Risiken (vgl. Deutscher Bundestag 2005). Besondere Armutsrisiken weisen Kinder von (langzeit-)erwerbslosen Eltern, in Ein-Eltern-Familien, in Familien mit Migrationshintergrund oder mit mehr als zwei Geschwistern auf. Weiterhin unterlie-

\begin{tabular}{|l|l|}
\hline Übersicht 1: Lebenslagedimensionen von Kinderarmut \\
\hline (1) Materielle Situation des Haushaltes („familiäre Armut ${ }^{\Perp}$ ) \\
\hline (2-5) Dimensionen der Lebenslage des Kindes \\
\hline (2) Materielle Versorgung des Kindes & $\begin{array}{l}\text { Grundversorgung } \\
\text { (zum Beispiel Wohnen, Nahrung, Kleidung) }\end{array}$ \\
\hline (3) „Versorgung “ im kulturellen Bereich & $\begin{array}{l}\text { Bildung } \\
\text { (zum Beispiel Arbeits-, Spiel- und Sprachverhalten) }\end{array}$ \\
\hline (4) Situation im sozialen Bereich & $\begin{array}{l}\text { Soziale Integration } \\
\text { (zum Beispiel Kontakte, soziale Kompetenzen) }\end{array}$ \\
\hline (5) Psychische und physische Lage & $\begin{array}{l}\text { Gesundheit } \\
\text { (zum Beispiel Gesundheitszustand, körperliche Entwicklung) }\end{array}$ \\
\hline Quelle: Zusammenstellung der Autorin. & \\
\hline
\end{tabular}

gen Mädchen und Jungen in Großstädten dort in benachteiligten Stadtteilen/Quartieren - einem höheren Risiko. Trotzdem leben in Deutschland in absoluten Zahlen die meisten armen Kinder in deutschen $\mathrm{Fa}$ milien und in ländlich geprägten Regionen. Einfache Zuschreibungen und Erklärungsmuster reichen offenkundig nicht aus.

\section{Der kindspezifische Armutsbegriff}

Armut ist mehr, als nur wenig Geld zu haben; Geld bleibt aber der Ausgangspunkt. Armut wird anhand der EU-Armutsdefinition bzw. auf der Grundlage des Konzepts der relativen Einkommensarmut bestimmt (Statistisches Bundesamt 2006). Daneben gelten hierzulande der Bezug von Sozialgeld bzw. Sozialhilfe sowie die Asylbewerberleistungen als Indikatoren. $\mathrm{Zu}$ diesem monetären Armutsbegriff kommen Aspekte der Unterversorgung in den Bereichen Bildung, sozialer Integration und Gesundheit hinzu. Armut beraubt Menschen ihrer materiellen Unabhängigkeit und damit auch der Fähigkeit, über existenzielle Fragen, über ihr „Schicksal“ selbst zu entscheiden. Sie wirkt mehrdimensional auf die gesamte Lebenslage eines Menschen und bestimmt dessen Gestaltungs-, Handlungs- und Entscheidungsspielräume. Armut ist demnach ein finanzieller Ressourcenmangel und eine defizitäre Lebenslage mit einer Vielzahl von materiellen wie immateriellen Folgen.

Um Kinderarmut oder - präziser - die Folgen von familiärer (Einkommens-)Armut bei Kindern zu erfassen, ist zunächst die Leitfrage bedeutsam: Was kommt (unter Armutsbedingungen) beim Kind an? Dabei ist Kinderarmut in vierfacher Hinsicht einzugrenzen und zu fokussieren: Es ist erstens vom Kind auszugehen; zweitens der familiäre Zusammenhang zu berücksichtigen und drittens ein mehrdimensionales Verständnis von Armut zugrunde zu legen, um Aussagen über die Entwicklungsund Teilhabechancen der betroffenen Kinder treffen zu können. Schließlich darf viertens Armut von Kindern nicht als Sammelbegriff für alle benachteiligenden Lebenslagen verwendet werden. Zur Bewertung der Entwicklungsbedingungen bzw. -möglichkeiten armer Kinder im oben verstandenen Sinn - vor allem im Vergleich zu ökonomisch besser gestellten Kindern - sind folgende Lebenslagedimensionen (Übersicht 1) zu berücksichtigen: materielle Haushaltssituation, materielle und kulturelle Versorgung, soziale Situation sowie physische und psychische Gesundheit des Kindes.

Für die Abgrenzung der Gruppe ,armer Kinder" bedeutet das: Von „Armut" wird immer und nur dann gesprochen, wenn „familiäre Armut" vorliegt, das heißt, wenn das Einkommen der Familie des Kindes bei maximal $50 \%$ resp. $60 \%$ des deutschen Durchschnittseinkommens liegt. Mädchen und Jungen, bei denen zwar Einschränkungen bzw. eine Unterversorgung in den oben genannten Lebenslagedimensionen (2-5) festzustellen sind, jedoch keine familiäre Armut vorliegt, sind zwar als benachteiligt zu bezeichnen, nicht jedoch als ,arm“.

Um einen umfassenden Blick auf die kindliche Lebenssituation zu erhalten, lassen sich die genannten vier Dimensionen in einem Lebenslageindex zusammenführen. Dieser umfasst drei Lebenslagetypen „Wohlergehen“, „Benachteiligung“ und „multiple Deprivation“: Von Wohlergehen wird dann gesprochen, wenn in Bezug auf die zentralen (Lebenslage-) Di-

\footnotetext{
2 Erste Ergebnisse wurden auf der 1. Armutskonferenz der Stadt Nürnberg vorgestellt. Die Dokumentation ist in Vorbereitung.
} 
Tabelle 1: Anteil der zehnjährigen Kinder mit Einschränkungen in den vier zentralen Lebenslagedimensionen und Armut 2003/04 - in \% -

\begin{tabular}{|c|c|c|c|c|}
\hline \multirow[t]{2}{*}{ Lebenslagedimension } & \multirow{2}{*}{$\begin{array}{l}\text { Arme Kinder } \\
\qquad(<50)\end{array}$} & \multicolumn{3}{|c|}{ Nicht-arme Kinder } \\
\hline & & $\begin{array}{l}\text { Prekärer } \\
\text { Wohlstand } \\
(50 \%-75)\end{array}$ & $\begin{array}{c}\text { Unterer } \\
\text { Durchschnitt } \\
(75 \%-100)\end{array}$ & $\begin{array}{c}\text { Oberer } \\
\text { Durchschnitt } \\
(>100)\end{array}$ \\
\hline Materielle Lage/Grundversorgung & 51,6 & 9,2 & 5,3 & 0,0 \\
\hline Kulturelle Lage & 37,7 & 19,0 & 9,5 & 3,6 \\
\hline Soziale Lage & 34,6 & 16,0 & 15,8 & 3,6 \\
\hline Gesundheitliche Lage & 25,8 & 23,3 & 21,1 & 8,4 \\
\hline$N=500$ & 159 & 163 & 95 & 83 \\
\hline
\end{tabular}

mensionen aktuell keine „Auffälligkeiten“ festzustellen sind, das Kindeswohl also gewährleistet ist. Eine Benachteiligung liegt gemäß Definition dann vor, wenn in einigen wenigen Bereichen aktuell „Auffälligkeiten" festzustellen sind. Das betroffene Kind kann in Bezug auf seine weitere Entwicklung als eingeschränkt beziehungsweise benachteiligt betrachtet werden. Von multipler Deprivation schließlich ist dann die Rede, wenn das Kind in mehreren zentralen Lebens- und Entwicklungsbereichen "auffällig" ist. Das Kind entbehrt in mehreren wichtigen Bereichen die notwendigen Ressourcen, die eine positive Entwicklung wahrscheinlich machen.

\section{Armutsfolgen - das Kinder- gesicht der Armut}

Mittlerweile kann ein profundes Forschungsmaterial zum Thema genutzt werden, sodass heute - im Gegensatz zu Ende der 1990er Jahre - kein allgemeines Erkenntnisdefizit (vgl. zuletzt Deutsches Kinderhilfswerk 2007) mehr gegeben ist: Die Armutsforschung weist nach, dass bereits im Vorschulalter arme Mädchen und Jungen erhebliche Unterversorgung erfahren. Die Gesundheitsforschung führt relevante Belege bereits für den Zeitpunkt der
Schwangerschaft der Frau und spätestens ab der Geburt des Kindes an (vgl. KIGGS 2007). Die Ernährungsforschung liefert ebenso wie die Bildungsforschung eindeutige Belege armutsbedingter Wirkungen ab frühem Kindesalter (Forschungsinstitut für Kinderernährung 2007; Konsortium Bildungsberichterstattung 2006).

In den AWO-ISS-Studien weisen rund $40 \%$ der armen, aber nur rund $15 \%$ der nicht-armen Kindern Mängel in der Grundversorgung (Wohnung, Kleidung, Ernährung) auf. Bis zum Ende der Grundschulzeit wächst der Anteil bei den armen Kindern auf über $52 \%$ an, dagegen sinkt er bei den nicht-armen Kindern gegen Null (Tabelle 1).

Ausdruck der Mangellage bei den 10Jährigen ist zum Beispiel, kein eigenes Zimmer zu haben und Einschränkungen bei der Kleidung und/oder beim Spielzeug hinnehmen zu müssen. Große Differenzen zwischen "arm" und "nicht arm" sind ebenso im kulturellen Bereich festzustellen: Arme Mädchen und Jungen haben weitaus weniger allgemeine und altersgemäße Lern- und Erfahrungsmöglichkeiten, zum Beispiel durch Vereinsmitgliedschaft, Teilnahme an freiwilligen Kursen inner- und außerhalb der Schule. Auch in der sozialen Lebenslage gibt es beträchtliche Unterschiede: Arme erhalten nicht nur einen begrenzten Raum zur sozialen Integration, ihnen wird zugleich die Möglichkeit zum breiten Erwerb sozialer Kompetenzen vorenthalten. Entsprechend zeigen sich erste Ansätze sozialer Devianz, also abweichenden Verhaltens. Dennoch: In den AWO-ISS-Studien sind zwischen $85 \%$ und $95 \%$ der Mädchen und Jungen, je nach Fragestellungen, nicht sozial auffällig. Das gilt auch für arme Kinder.

Gleichwohl ist der Trend eindeutig: Arme und nicht arme Kinder nehmen einen vollkommen unterschiedlichen und immer weiter divergierenden Entwicklungsverlauf, wobei für Erstere der „Fahrstuhl“ eher nach unten und für Letztere eher nach oben geht. Je früher und je länger ein Kind Armutserfahrungen macht, desto gravierender sind die Folgen für seine gegenwärtige Lebenssituation und seine Zukunftschancen. Das Spektrum, aber auch die zum Teil gegenläufige Verteilung der Lebenslagetypen, zeigt sich im Vorschulalter genauso wie im späten Grundschulalter ( $\mathrm{Ta}$ belle 2). Die Spreizung zwischen Wohlergehen und multipler Deprivation ist Ergebnis von Sozialisationsprozessen und -bedingungen ab der Geburt; sie ist zugleich die Grundlage für den weiteren kindlichen Entwicklungsprozess.

Die kindbezogene Armutsforschung belegt zweierlei: Zum einen ist eine hohe Dynamik in den Entwicklungsverläufen der Kinder erkennbar, das heißt Bewegungen aus der Armut bzw. Deprivation und in die Armut bzw. Deprivation kommen häufig vor. Es gilt also nicht ,einmal arm - immer arm" und „einmal multipel depriviert - immer multipel depriviert“. Zum anderen finden sich stark divergierende Lebensund Entwicklungsverläufe. Unter den Armen überwiegen negative Verläufe, das heißt: Zunahme von Auffälligkeiten in den Lebenslagen und Wechsel des Lebenslagentyps, z. B. von Benachteiligung nach multipler Deprivation. Bei den nicht-armen Kindern dominiert eine gefestigte positive Entwicklung, so beispielsweise Verbleib im Wohlergehen.

\section{Schutzfaktoren können Armutsfolgen auffangen}

Armut ist der größte Risikofaktor für die kindlichen Lebenschancen. Über welche individuellen, sozialen und kulturellen Ressourcen zur Bewältigung ihrer Auswirkungen ein Kind verfügt und welche Hand- 
lungsstrategien respektive welches Bewältigungshandeln es bei Belastungen entwickelt, hängt entscheidend von der Lebenslage ab und baut auf einer Vielzahl von intervenierenden (persönlichen, familiären und außerfamiliären) Variablen auf. Ebenso sind außerfamiliäre Angebote und Sozialisationsinstanzen, wie Kindertagesstätten und der unmittelbare Lebensraum, entscheidende Einflussfaktoren. Dort kann sowohl Einfluss auf die Entwicklung von Resilien $z^{3}$ wie auch auf die Entstehung und Unterstützung eines förderlichen Umfelds genommen werden (Richter 2006; DGgKV 2007; Zander 2008). Dies alles können Schutzfaktoren sein.

Die AWO-ISS-Studien belegen die Existenz zahlreicher solcher wirkungsvoller Schutzfaktoren. Dazu zählen unter anderem das (Alltags-)Bewältigungshandeln von Eltern, das Erlernen von positiven Handlungsstrategien und eine gelingende, weil geförderte, soziale Integration in Peergroups sowie das soziale und schulische Umfeld. Es besteht ein deutlicher Zusammenhang zwischen der Gesamtzahl von Schutzfaktoren und dem Lebenslagetyp. Im Wohlergehen aufwachsende Kinder weisen im Mittel mehr Schutzfaktoren $(13,7)$ auf als multipel deprivierte $(11,3)$ (Holz et al. 2006, S. 191-200). Es besteht weiterhin ein Zusammenhang zwischen der Zahl der verfügbaren Schutzfaktoren und dem kindlichen Entwicklungsverlauf: Kinder, die eine gefestigte positive Entwicklung erleben, können auf mehr Schutzfaktoren zurückgreifen als permanent multipel deprivierte. Das weist darauf hin, dass es bei Interventionen bezüglich der Rahmenbedingungen des kindlichen Aufwachsens zu einer unmittelbaren Wirkung auf die Lebenslage und das Bewältigungsverhalten von Minderjährigen kommt. Es sind weder allein individuell erworbene Kompetenzen noch "Selbstheilungskräfte“ (der Familie), sondern soziale Förderung, Ausgleich und Umverteilung, die den Betroffenen wirkliche Zukunftschancen eröffnen.

\section{Kindbezogene Armutsprävention}

Da Armut ein gesellschaftliches Phänomen struktureller Art ist, kann Armutsbekämpfung (d.h. Vermeidung oder Verminderung) nur durch eine soziale Gegensteue- rung erfolgen. Bei der Frage „Kinderarmut" sind stets die Lebenssituation und die Handlungsmöglichkeiten der Eltern einzubeziehen. Fehlen innerhalb der Familie die materiellen Ressourcen sowie die elterlichen Kompetenzen oder Potenziale, um den eigenen Kindern ein Aufwachsen im Wohlergehen zu ermöglichen, dann ist es für diese umso wichtiger, eine Kompensation oder Ergänzung durch außerfamiliäre Förderung zu erhalten. Es geht um öffentliches Engagement in zweierlei Hinsicht: Zum einen im Sinne einer sozialen Gegensteuerung mit dem Fokus auf „strukturelle Armutsprävention“ durch Gestaltung bzw. Veränderung von Verhältnissen und Rahmenbedingungen; zum anderen um soziale Gegensteuerung mit dem Fokus auf „individuelle Förderung und Stärkung " durch Gestaltung bzw. Veränderung von individuellem Verhalten.

Armutsprävention als gesellschaftliche Verpflichtung und sozialstaatlicher Auftrag wird entscheidend durch Politik und Verwaltung bestimmt. Sie beinhaltet das Aktivwerden auf unterschiedlichen Handlungsebenen und vereint in sich politische, soziale, pädagogische und planerische Elemente. Sie umfasst Maßnahmen der Gegensteuerung durch Gestaltung von Rahmenbedingungen, aber auch über die Bereitstellung sozialer Ressourcen und die Förderung integrativer Prozesse. Akteure sind sowohl die politisch Verantwortlichen auf kommunaler, Landes-, Bundes- und EU-Ebene, als auch Organisationen, Institutionen und die dort tätigen Fachkräfte sowie die Bürger und Bürgerinnen selbst.

Kindbezogene Armutsprävention stellt einen theoretischen und praktischen Handlungsansatz dar, der aus der Kindperspektive heraus auf positive Lebens- und Entwicklungsbedingungen für Kinder heute und morgen hinwirkt. Leitorientierung ist die Sicherung eines „Aufwachsens im Wohlergehen" für alle Kinder und speziell für arme. Hauptziel ist es, kindspezifische Armutsfolgen zu vermeiden respektive zu begrenzen, aber auch Ursachen auf Seiten der Eltern, der Familien und des Umfeldes positiv zu beeinflussen. Ein solches öffentliches Engagement für (arme) Kinder kann in drei Richtungen erfolgen:

(1) Indirekt durch eltern- bzw. familienbezogene Maßnahmen, wie z. B. Arbeitsmarktpolitik, Förderung der Vereinbarkeit von Familie und Erwerbstätigkeit, Erweiterung der sozialen Sicherung usw.;
(2) Wiederum indirekt, durch sozialraumbezogene Maßnahmen, wie z. B. Stadtentwicklung im Sinne von besonderen Aktivitäten in sozial belasteten Quartieren oder auch generell als kinderfreundliche Kommune usw.;

(3) Direkt durch kindbezogene Maßnahmen, wie beispielsweise frühe Förderung, Gesundheitsvorsorge und Lernmittelfreiheit in und Ausbau von Krippen, Kindertagesstätten, Horten und Schulen und anderes mehr.

Es gilt die Resilienz und damit die Widerstandskräfte des Einzelnen zu fördern (Verhalten), aber auch, durch die Ausweitung struktureller Armutsprävention für bessere Lebens- und Entwicklungsbedingungen zu sorgen. Beides bedingt sich gegenseitig und kennt dennoch eigene Schwerpunkte.

Die Umsetzung struktureller Armutsprävention für Kinder richtet sich an alle Staatsebenen, besonders aber an die Kommunen, denn das lokale Geschehen bildet den unmittelbaren Bezugspunkt zu Leben und Entwicklung eines Kindes. Für eine solche Armutsprävention ist das Netzwerk, gebildet durch alle relevanten Akteure aller betroffenen Bereiche auf der jeweiligen Ebene, die geeignete Struktur. Kennzeichnend dafür sind die ressortübergreifende Verknüpfung und das abgestimmte Handeln der Akteure im Rahmen von Gesetzgebungs- und Verwaltungsverfahren. Eine weitere Struktur der Armutsprävention ist die Präventionskette, wiederum gebildet von allen öffentlichen und gesellschaftlichen Akteuren, die für das Erreichen des jeweiligen Präventionsziels verantwortlich sind. Sie ist biografisch angelegt und darauf ausgerichtet, jedem Kind eine fördernde Begleitung - je nach Bedarf und zu jedem möglichen Zeitpunkt - von der Geburt an bis zum erfolgreichen Berufseinstieg zuzusichern.

Netzwerk und Präventionskette sollen sicherstellen, dass die Kernpunkte einer kindbezogenen Armutsprävention gewährleistet werden. Diese sind mit Bezug auf die Art des Eingriffs:

- Beginn der Prävention zum frühestmöglichen Zeitpunkt;

\footnotetext{
3 Resilienz bezeichnet die individuelle Fähigkeit, schwierige Lebenssituationen unbeschadet zu überstehen.
} 
- Eltern und Familie sind zentrale Adressaten;

- Berücksichtigung der Lebenswelt der Kinder (Kindertagesstätte, Schule, Nachbarschaft, Stadtteil usw.);

- umfassende, finanziell zugängliche und emotional ansprechende außerfamiliäre Angebote für Bildung, Erziehung, Betreuung und Beratung als zentrale Garanten für den Zugang zu sozialen Ressourcen.

Als Ziele der Armutsprävention sollen die Strukturformen Folgendes erreichen:

- Sicherung der existenziellen Lebensund Entwicklungsmöglichkeiten des Kindes;

- Förderung von Teilhabe, Integration, Bildung und Gesundheit;

- Förderung der Potenziale und Ressourcen des Kindes;

- Stärkung der Kinder durch das Erlernen von problemlösendem Bewältigungshandeln;

- Verhinderung oder Bewältigung von kindlichen Entwicklungsdefiziten.

Für die Akteure der Armutsprävention bedeutet dies, dass

- die kommunale Kinder- und Jugendhilfe aktiv die strategische Federführung ausübt;

- die Träger und Fachkräfte des Bildungs-, Sozial- und Gesundheitswesens mitverantwortlich für bedarfsgerechte und passgenaue Förderangebote sind;

- Politik und Verwaltung auf kommunaler, Landes- und Bundesebene primär präventiv wirkende gesellschaftliche Rahmenbedingungen schaffen.

Diese Elemente - als Teilziele auf lokaler und auf institutioneller Ebene formuliert lassen sich durch Maßnahmen, Angebote und Projekte operationalisieren sowie auf ihre nachhaltige Wirkung hin überprüfen.

Die dargestellte Form kindbezogener Armutsprävention geht weit über Sonntagsreden und kurzatmige Einzelprojekte hinaus. Sie ist eine breit angelegte Strategie sowie ein permanenter Prozess und spiegelt die „öffentliche Verantwortung“ unserer Gesellschaft für die Problematik wider. Sie ist Teil sozialstaatlichen Handelns mit ausdrücklicher Ausrichtung auf Kinder.

\section{0̈ \\ "Öffentliche Verant- wortung" ist staatliches Handeln auf allen Ebenen}

Der Kommune kommt als Lebensort von Kindern immer die verantwortliche Gesamtsteuerung einer kindbezogenen Armutsprävention zu, wie es im SGB VIII verankert ist. Hier wird der konkrete Lebensalltag von Kindern gestaltet, hier zeigt sich der (Miss-)Erfolg eines gesamtgesellschaftlichen Präventionsnetzwerkes. Entscheidend aber ist, dem lokalen Handeln und dem Engagement Einzelner eine mindestens so breite Bewegung auf Landes- und Bundesebene zum einen vorausgehen und zum anderen folgen zu lassen. Dort wird über die strukturellen Rahmenbedingungen insgesamt entschieden. Dort sind Antworten auf folgende Fragen zu geben und rasch wirkungsvolle Umsteuerungen vorzunehmen:

(1) Wie wird eine eigenständige armutsfeste Grundsicherung für Kinder als Teil von Kinderrechten realisiert? Das betrifft z. B. Fragen finanzieller Zuwendungen wie Kindergeld, Kinderzuschlag, Kinderfreibeträge und Ehegattensplitting, aber auch des Erwerbseinkommens im Sinne von armutsfesten Mindestlöhnen und der Vermeidung von Niedriglöhnen oder „working poor“"

(2) Wie werden Eltern bzw. Familien in ihrer Verantwortung für ihre Kinder gestärkt und arme Eltern um einen Teil ihrer Überbelastung entlastet? Hier stellen sich beispielsweise Fragen zum Ausbau und zur Qualität der sozialen Dienstleistungen, vor allem für armutsgefährdete Gruppen, zur Bildung und Weiterbildung gering qualifizierter Erwachsener und zum lebenslangen Lernen für bildungsferne Bevölkerungsgruppen.

(3) Wie wird ein hohes Qualifikationsniveau bei (armen) Kindern und Jugendlichen erreicht? Dabei stehen Fragen der schulischen Breitenförderung auf hohem Niveau im Vordergrund. Diese ist Voraussetzung für die Umsetzung des Bildungsund des sozialen Integrationsauftrags von Kindertagesstätten und Schulen.
(4) Wie wird die Teilhabe armer Kinder an den gesellschaftlichen Ressourcen gesichert? Das betrifft z. B. die Nutzung von und den Zugang zu allgemeiner Infrastruktur, zu Gesundheitsförderung und präventiver Kinder- und Jugendhilfe sowie die Teilhabe an Kultur- und Freizeitangeboten.

(5) Wie wird eine Lebenswelt für Kinder geschaffen, die auf soziale Integration und Förderung aufbaut? Hier stellen sich Fragen der Sicherung von Daseinsvorsorge durch Kommunen, zur integrierten Stadt(entwicklungs-)planung und sozialen Wohnungspolitik, zur Schaffung kindgerechter Lebenswelten, aber auch zur allgemeinen Sozialberichterstattung als einem Basisinstrument zur Armutsprävention.

\section{Resümee}

Kinderarmut existiert in erheblichem Umfang und verschwindet nicht ohne aktives Handeln. Strukturelle Ursachen dieses gesellschaftlichen Massenphänomens erfordern eine soziale Gegensteuerung, die auf Armutsprävention abzielt. Dabei sind immer Maßnahmen auf zwei Ebenen zu realisieren und in ihrem genuinen Zusammenhang zu berücksichtigen: Veränderung von ursächlichen gesellschaftlichen Verhältnissen bzw. Rahmenbedingungen und Veränderung von problemförderndem Verhalten des Einzelnen. Im Focus steht das Kind mit seinen Lebensbezügen. Das „Aufwachsen im Wohlergehen aller Kinder" ist die Leitorientierung einer kindbezogenen Armutsprävention. Dies ist zugleich die kindspezifische Ausgestaltung des sozialstaatlichen Auftrages. Darin eingebunden sind alle kindlichen Entwicklungsbereiche, alle öffentlichen Handlungsfelder sowie alle Institutionen, Ressorts und Professionen. In konzeptioneller Hinsicht bildet der „Nationale Aktionsplan: Für eine kindgerechte Welt 2005-2010“ (BMFSFJ 2005b) als ein Ausdruck der Umsetzung der UNKinderrechte durch die Bundesregierungbereits seit 2005 de jure eine sehr gute Grundlage für staatliches Handeln. Die rasche Umsetzung in die Praxis ist der entscheidende Schlüssel für eine positivere Zukunft. 
Bundesagentur für Arbeit (BA) (2007): Grundsicherung für Arbeitssuchende - SGB II. Monatsbericht November 2007, Nürnberg, online: www.pub.arbeitsamt.de/hst/services/statistik/000000/html/start/ monat/aktuell.pdf

Becker, I. (2006): Armut in Deutschland: Bevölkerungsgruppen unterhalb der Alg II-Grenze. Arbeitspapier 3 des Projekts "Soziale Gerechtigkeit" der J. W. Goethe-Universität Frankfurt/Main, online: www.boeckler.de/ pdf fof/S-2006-863-4-3.pdf

Bundesministerium für Familien, Senioren, Frauen und Jugend (BMFSFJ) (Hrsg.) (2005a): Zwölfter Kinder- und Jugendbericht über die Lebenslagen junger Menschen und die Leistungen der Kinder- und Jugendhilfe, Berlin, online: www.bmfsfj.de/RedaktionBMFSFJ/Abteilung5/Pdf-

Anlagen/zwoelfter-kjb, property=pdf.pdf

Bundesministerium für Familien, Senioren, Frauen und Jugend (BMFSFJ)

(Hrsg.) (2005b): Nationaler Aktionsplan: Für eine kindgerechtes Deutschland 2005-2010, Berlin, online: www.bmfsfj.de/bmfsfj/generator/ Publikationen/nap/nationaler-aktionsplan-fuer-ein-kindergerechtesdeutschland-2005-2010.html

Bundesministerium für Bildung und Forschung (BMBF) (Hrsg.) (2007): Bildung auf einen Blick 2007, Bonn

Deutsche Gesellschaft gegen Kindesmisshandlung und -vernachlässigung (DGgKV) (Hrsg.) (2007): Themenheft 1, „Resilienz, Ressourcen, Schutzfaktoren: Kinder, Eltern und Familien stärken“, Lengerich Deutscher Bundestag (2005): Lebenslagen in Deutschland. Der Zweite Armuts- und Reichtumsbericht der Bundesregierung, Berlin (BT-Drucksache 15/5015)

Deutscher Bundestag (2007): Antwort der Bundesregierung auf die Kleine Anfrage "Überwindung der "Hartz IV" -Abhängigkeit von Kindern und Eltern durch den Kinderzuschlag“" Berlin (Bt-Drucksache 16/7586), online: http://dip21.bundestag.de/dip21/btd/16/075/1607586.pdf.

Deutsches Kinderhilfswerk (Hrsg.) (2007): Kinderreport 2007, Freiburg Deutsches Institut für Wirtschaftsforschung (DIW) (2007): Nach der Einführung von Arbeitslosengeld II: Deutlich mehr Verlierer als Gewinner unter den Hilfeempfängern, online: www.diw.de/documents/publikationen/ 73/76990/07-50-1.pdf

Forschungsinstitut für Kinderernährung (FKE) (2007): Arbeitslosengeld II reicht nicht für gesunde Kinderernährung, online: www.fke-do.de/ content.php?seite $=$ seiten $/$ inhalt. php\&details $=854$
Hock, B./Holz, G./Simmedinger, R./Wüstendörfer, W. (2000): „Gute Kindheit - Schlechte Kindheit?" , Armut und Zukunftschancen von Kindern und Jugendlichen in Deutschland, Frankfurt am Main

Holz, G./Richter, A./Wüstendörfer, W,/Giering, D. (2006): Zukunftschancen von Kindern? Wirkung von Armut bis zum Ende der Grundschulzeit, Frankfurt am Main

KIGGS im Robert Koch-Institut (2007): Studie zur Gesundheit von Kindern und Jugendlichen in Deutschland. Gesamtüberblick und Darstellung erster Ergebnisse, online: www.kiggs.de/experten/fachbeitraege/index. html. sowie http://www.kiggs.de/experten/downloads/dokumente/ BG_45_852_858.pdf

Konsortium Bildungsberichterstattung (2006): Bildung in Deutschland. Ein indikatorengestützter Bericht mit einer Analyse zu Bildung und Migration, Bielefeld

Meier, U./Preuße, H./Sunnus E.-M. (2003): Steckbriefe von Armut, Haushalte in prekären Lebenslagen, Wiesbaden

Richter, A. (2006): Was brauchen arme Kinder? - Resilienzförderung und Armutsprävention, in: Holz, G. (Hrsg.): Armut bei Kindern, KiTa spezial 4, S. 9-14

Statistisches Bundesamt (2006): Armut und Lebensbedingungen. Ergebnisse aus LEBEN IN EUROPA für Deutschland 2005 - Pressexemplar, Wiesbaden, online: www.forschungsdatenzentrum.de/bestand/eu-silc/ $\mathrm{fdz}$ eu-silc-bericht.pdf

Statistisches Bundesamt (2007): Statistisches Jahrbuch 2007 für die Bundesrepublik Deutschland, Wiesbaden, online: www.destatis.de/ jetspeed/portal/cms/Sites/destatis/Internet/DE/Navigation/ Publikationen/ Querschnittsveroeffentlichungen/Jahrbuch,templateld $=$ renderPrint.psml_nnn=true.

Schröder, P. M. (2007): Kurzmitteilung „Nicht erwerbsfähige Hilfebedürftige im Alter unter 15 Jahren“, Bremen, Stand: 15. August, online: www.arbeitnehmerkammer.de/Sozialpolitik/doku/1_aktuell/ticker/2007/ 2007_08_15_biaj.pdf

Walper, S. (2005): Tragen Veränderungen in den finanziellen Belastungen von Familien zu Veränderungen in der Befindlichkeit von Kindern und Jugendlichen bei?, in: Zeitschrift für Pädagogik 2, S. $170-191$

Zander, M. (2008): Armes Kind - starkes Kind?, Die Chance der Resilienz, Wiesbaden 